\title{
Die Wahrnehmung der Litauer durch den Deutschen Orden um 1400 und die Rolle des kollektiven Gedächtnisses
}

\section{The Perception of the Lithuanians by the Teutonic Order around 1400 and the role of collective memory}

Sebastian Kubon / sebastian.kubon@uni-hamburg.de Universität Hamburg, Arbeitsbereiche Mittelalterliche Geschichte \& Public History, DE

\begin{abstract}
Not only the perception of the contemporary Lithuanians and Vytautas by Konrad von Jungingen (1393-1407) and the Teutonic Order, but also the Order's memory of the earlier actions of the Lithuanians under King Mindaugas were the background against which the policy of the beginning 15th century was conducted. There is a nexus between memory, perception and politics. The Order still perceived the Lithuanians around 1400 as pagans. The memory studies, especially the central ideas of Maurice Halbwachs' classical theories on collective memory and the "cadres sociaux" provide a plausible explanation.
\end{abstract}

\section{Keywords}

the Teutonic Order; Lithuanians; Konrad von Jungingen; Collective Memory; Vytautas 
Ein Brief von Konrad von Jungingen an den Generalprokurator des Ordens in Rom, den Deutschmeister und den Landkomtur von Böhmen wirft ein Schlaglicht auf die Wahrnehmung des Hochmeisters bzgl. der Litauer und ihrer Christianisierung im Jahr 1396. Der Inhalt des Briefes betrifft hier hauptsächlich Herzog Vytatutas von Litauen, den Cousin des Königs Wladyslaw II. Jagiello von Polen-Litauen. Es heißt hier: ${ }^{1}$ Dorumb, das got nicht gebe, ab eyn umslag geschege, als vor ofte gescheen ist, von im [Vytautas; S.K.] und sienen vorvarn, als von konig Mindow von Littowen, das sich der orden sien deste bas mochte dirweren des umslages adir abkerunge. Der Hochmeister betont, dass Vytautas und seine Vorfahren, wie König Mindaugas von Litauen, oft vom Christentum abgefallen und zum Heidentum zurückgekehrt seien. Der Orden rief also nicht nur die zeithistorischen Kämpfe mit den Litauern in Erinnerung, sondern auch die nur temporäre Christianisierung unter Mindaugas, die um das Jahr 1250 und damit vor ca. 150 Jahren stattgefunden hatte! ${ }^{2}$ Offensichtlich gab es eine Verbindung zwischen der Erinnerung an vergangene Ereignisse und der Wahrnehmung der Gegenwart und ihrer spezifischen Interpretation. Dies dürfte annehmen lassen, dass die Erinnerung an historische Ereignisse eine entsprechende Politik des Hochmeisters Konrad in der Gegenwart präfiguriert hat.

Obgleich der Titel einen umfassenden Ansatz verspricht, soll es im Folgenden doch vielmehr darum gehen, ein paar einzelne Textstellen zur Wahrnehmung der Litauer hauptsächlich in der Zeit des Hochmeisters Konrad von Jungingen (1393-1407)³ genauer unter die Lupe zu nehmen. Diese finden sich dafür allerdings in Quellen, die entweder bislang nicht die Aufmerksamkeit erhalten haben, die sie verdienen, oder die - viel gravierender - bislang aus einer zu modernen Perspektive interpretiert wurden. Dies geschieht in der Hoffnung, einen Erklärungsansatz für eine der zentralen und umstrittensten Fragen zur Geschichte des Deutschordenslandes Preußen um 1400 anzubieten: ${ }^{4}$ Warum hat der Deutsche Orden damals noch seine Kämpfe gegen Litauen

1 Die Berichte der Generalprokuratoren des Deutschen Ordens an der Kurie (= BGDO). Bd. I.: Die Geschichte der Generalprokuratoren von den Anfängen bis 1403. Bearb. K. Forstreuter. Göttingen 1961, Nr. $246=$ Berlin, Geheimes Staatsarchiv Preußischer Kulturbesitz, XX. Hauptabteilung, Ordensfoliant (= OF) 2c, S. 69-72. Vgl. zu diesem Brief auch Kubon, Sebastian: Der Vertrag von Sallinwerder (1398) und sein Bruch aus der Sicht des Deutschen Ordens - mit einem Quellenanhang. In: Der Bruch des Vertrags. Hg. v. G. Jostkleigrewe. Zeitschrift für Historische Forschung. Beiheft 55, 2018, S. 309-336, hier S. 316-317.

2 Zur Zeit von Mindaugas vgl. KIAUPA, Zigmantas: The History of Lithuania before 1795. Vilnius 2000, S. 54-68.

3 Zur Außenpolitik des Hochmeisters Konrad von Jungingen nicht zuletzt gegenüber Litauen und Samaiten Kubon, Sebastian: Die Außenpolitik des Deutschen Ordens unter Hochmeister Konrad von Jungingen (1393-1407). Göttingen 2016 (= Nova Mediaevalia 15) sowie auf einem abstrakteren Niveau in aller Kürze DERS.: Die Grundzüge der Außenpolitik des Deutschordenshochmeisters Konrad von Jungingen (1393-1407). Blätter für deutsche Landesgeschichte 152, 2016, S. 85-97. Hier finden sich jeweils schon Beobachtungen zur Wahrnehmung der Litauer und Samaiten durch Konrad von Jungingen versammelt. In KuBon, S.: Sallinwerder, steht die Vertragsbrüchigkeit der Litauer in der Wahrnehmung des Ordens und der Umgang damit im Mittelpunkt der Betrachtungen. Ibidem, S. 328, wurde auf die Bedeutung der in dieser Studie untersuchten Fragstellung schon hingewiesen.

4 An Gesamtdarstellungen zur Geschichte des Deutschen Ordens bzw. des Ordenslandes Preußen herrscht kein Mangel mehr: vgl. SARnowsky, Jürgen: Der Deutsche Orden. München 2007; Biskup, Marian - Czaja, Roman (Hg.): Państwo zakonu krzyżackiego w Prusach. Wtadza i spoteczeństwo. Warszawa 2008; GouguenheIM, Sylvain: Les chevaliers teutoniques. Paris 2007. 
und die Litauer weitergeführt, ${ }^{5}$ obgleich deren offizieller Übertritt zum Christentum im Rahmen der polnisch-litauischen Union ${ }^{6} 1386$ eigentlich schon vor über einer Dekade stattgefunden hatte?

Es geht daher im Folgenden erstens darum anzudeuten, dass nicht nur die Wahrnehmung der mehr oder weniger zeitgenössischen Litauer durch Konrad von Jungingen (1393-1407) und den Deutschen Orden, sondern auch die Erinnerung des Ordens an die früheren Taten der Litauer der Hintergrund war, vor dem die aktuelle Politik des beginnenden 15. Jahrhunderts betrieben wurde. Das legt schon das Eingangszitat nahe, in dem sich ein Zusammenhang zwischen Erinnerung, Wahrnehmung und Politik spiegelt. An dieser Stelle können jedoch keine endgültigen Resultate präsentiert werden. Vielmehr sollen erste Überlegungen zu dieser intrikaten Frage zur Diskussion gestellt werden - rein auf Basis schriftlicher Quellen. Zweitens soll darauf hingewiesen werden, dass es vielleicht die Theorien der kulturwissenschaftlichen Gedächtnisforschung ${ }^{7}$ sind, $^{2}$ die für diese Situation eine plausible Erklärung anbieten können und die daher in extenso rezipiert werden sollten. In dieser Skizze kann es einzig darum gehen, anhand von einigen zentralen Gedanken der klassischen Studien von Maurice Halbwachs ${ }^{8}$ zum kollektiven Gedächtnis und den „cadres sociaux“ bzw. der sozialen Bedingtheit von Erinnerung das mögliche Potential für die Untersuchung der Situation im Deutschordensland Preußen um 1400 exemplarisch hervorzuheben und weitere vertiefte Studien anzuregen.

Kurz zur Vorgeschichte der Regierungszeit Konrads, da hier letztlich die relevanten Ereignisse stattgefunden haben, die eine Änderung der politischen ,Großwetterlage dieser Region bedeuten sollten: ${ }^{9}$ Es war das Jahr 1386, das mit der Gründung der polnisch-litauischen Union als großer Wendepunkt auch in der Geschichte Preußens gelten muss. Der litauische Großfürst Jogaila trat zum Christentum über, heiratete die polnische Thronerbin Jadwiga und wurde polnischer König unter dem Namen Wladyslaw II. Jagiello. Nun konnte man Litauen als Teil der christlichen Oikumene betrachten. Der Orden verlor damit seine Daseinsberechtigung in dieser Gegend. So zumindest interpretierte die polnisch-litauische Seite die neue Situation. Der Orden sah dies jedoch anders. Die Deutschordensritter behandelten die Christianisierung nur als eine scheinbare und setzten den Kampf gegen die Litauer daher fort. Alles in allem war dies eine seltsame Situation, die eine Erklärung verlangt. Nach der opinio communis der Sekundärliteratur,

5 Paravicini, Werner: Die Preussenreisen des europäischen Adels. 2 Bde. Sigmaringen 1989-1995 (= Beihefte der Francia 17/1-2).

$6 \quad$ BŁAszczyk, Grzegorz: Dzieje stosunków polsko-litewskich od czasów najdawniejszych do wspótczesności. I: Trudne poczatki. Poznań 1998. Ders.: Dzieje stosunków polsko-litewskich. II: Od Krewa do Lublina. Poznań 2007.

7 Als einführende Zusammenfassung zu den Ansätzen der kulturwissenschaftlichen Gedächtnisforschung vgl. ErLl, Astrid: Kollektives Gedächtnis und Erinnerungskulturen. Eine Einführung. Stuttgart ${ }^{32017}$ (hier auch umfangreiche Literaturhinweise) oder Pethes, Nicolas: Kulturwissenschaftliche Gedächtnistheorien zur Einführung. Hamburg 2008.

8 Vgl. besonders die Schriften von Halbwachs, Maurice: Das Gedächtnis und seine sozialen Bedingungen. Berlin 62019 [frz. Original 1925] und DERs.: Das kollektive Gedächtnis. Frankfurt am Main 1991 [frz. Edition 1950].

9 Der Forschungsstand ist im Detail zusammengefasst bei Kubon, S.: Außenpolitik, S. 53-57 mit entsprechenden Literaturnachweisen. 
die sich besonders pointiert bei Hartmut Boockmann widerspiegelt, fuhr der Orden wider besseres Wissen fort, die Litauer als Heiden zu behandeln und zu bekämpfen. ${ }^{10}$ Das Pochen auf Christianisierung ist demnach nur ein Vorwand gewesen, um Land in Litauen, so besonders Samaiten ${ }^{11}$ (Niederlitauen), das zumindest zeitweise unter Konrad von Jungingen in Besitz genommen werden konnte, zu erobern und den Machtbereich zu erweitern. ${ }^{12}$ Der Orden habe den Übertritt zum Christentum schlicht nicht wahrhaben wollen und betrieb weiterhin Expansion unter dem Deckmantel des Heidenkampfes - so die bis vor kurzem unhinterfragte Auffassung dieser Situation. Damit wurde das Handeln des Deutschen Ordens gegenüber der polnisch-litauischen Union also bislang nur rein machtpolitisch erklärt.

Welcher Zusammenhang, um zum ersten Aspekt zu kommen, besteht aber nun zwischen Wahrnehmung, Erinnerung und Politik des Hochmeisters bzw. des Ordens hinsichtlich Litauens und der Litauer? Erst kürzlich wurden die der Politik gegenüber Litauen und Samaiten zugrundeliegenden Motive Konrads von Jungingen an verschiedenen Orten umfänglich untersucht. ${ }^{13}$ Das Ergebnis war, dass die Kämpfe militärischer und diplomatischer Art gegen die Litauer und Samaiten (Niederlitauer) nicht primär als Versuche der zielgerichteten territorialen Expansion gewertet werden können, wie es in der oben skizzierten opinio communis getan wurde, sondern vornehmlich als Versuche der Christianisierung - dieses Mal allerdings unter den Bedingungen des Deutschen Ordens. ${ }^{14}$ Es ist sehr wahrscheinlich, dass hier keine Propaganda zu sehen ist. Hochmeister Konrad von Jungingen nahm die Litauer offensichtlich immer noch und nicht wider besseres Wissen als nur scheinbare Christen wahr.

Das ist nun dann doch eine recht unerwartete Erkenntnis, existierte die polnisch-litauische Union eben immerhin schon seit ca. zehn Jahren. Wie also kann man diese doch eher überraschende Wahrnehmung des Hochmeisters erklären? Warum hat der Hochmeister die sich geänderten Rahmenbedingungen nicht erkannt bzw. nicht erkennen können? Es gibt drei einschlägige Quellenstellen, die hier in den Mittelpunkt der Analyse gestellt werden sollen, die zeigen, dass Hochmeister Konrad von Jungingen die Litauer tatsächlich weiterhin als nur scheinbare Christen wahrgenommen hat aufgrund der - und das ist die These, deren Stimmigkeit hier wahrscheinlich gemacht werden soll - im Ordensland Preußen vorherrschenden Erinnerung sowohl an die mehr oder weniger zeitgenössischen Christianisierungsversuche als auch an die gescheiterten Versuche

10 Boockmann, Hartmut: Der Deutsche Orden. Zwölf Kapitel aus seiner Geschichte. München 1981, S. 153-155, 172, passim; DERs.: Johannes Falkenberg, der Deutsche Orden und die polnische Politik. Untersuchungen zur politischen Theorie des späteren Mittelalters. Göttingen 1975 (= Veröffentlichungen des Max-Planck-Instituts für Geschichte 45), S. 76-77.

11 Im Detail zum ,Erisapfel‘ Samaiten vgl. Kubon, S.: Außenpoltitik, S. 58-192 (hier ist auch die ältere Literatur versammelt).

12 Werner Paravicini spricht explizit von einem „Eroberungskrieg“ gegenüber Litauen, PAravicinI, W: Preussenreisen, I, S. 23, 24.

13 Vgl. Kubon, S.: Außenpolitik; Ders.: Sallinwerder; DERS.: Grundzüge.

14 Zudem war die Expansion nach Samaiten von Vytautas aufgedrängt und wurde erst recht nicht zielgerichtet von Konrad von Jungingen betrieben, der vielmehr ein Getriebener der Zumutungen von außen war; vgl. Kubon, S.: Außenpolitik, S. 187-192, 325-333. 
früherer Zeiten, die die Schablone bildeten für die Wahrnehmung der Litauer zur Zeit Konrads von Jungingen.

Die erste Quellenstelle findet sich in einem offenen Brief des Deutschen Ordens an die westliche Öffentlichkeit in einer deutschen und einer lateinischen Fassung. Der Brief aus dem Jahre 1403 enthält zahlreiche Hinweise auf die Ordenswahrnehmung zum Stand der Christianisierung und der Religion der Litauer. Der Orden wollte damit die Beschwerden der polnisch-litauischen Seite, die allerdings nur aus der Antwort des Hochmeisters hervorgehen, zurückweisen. Diese hatte den Orden angeklagt, die Hauptkirchen und auch die anderen Kirchen in Litauen und Russland zu zerstören, die unter hohen Kosten gebaut worden seien. Es heißt in der Quelle auf deutsch (mit der Entsprechung der lateinischen Fassung in Klammern): ${ }^{15}$ unrechter beschuldigunge czihet her uns, wie wir die houptkirchen (Ecclesiarum ... kathedralium) und ander kirchen yn synen landen Littouwen und Rußen tylgen, vorterben und heeren, die her gebuwet hat, und wedirbuet mit großen kosten. Der Hochmeister antwortete darauf, dass es keine aufwändig gebauten Kirchen (kostlichen kirchen) gebe. Wen es Kirchen in Litauen gebe, so seine Antwort weiter, dann glichen sie einem ärmlichen Wohnhaus (eyme armen wonhuße). Hartmut Boockmann hat schon 1975 auf diesen Brief explizit hingewiesen. Letztlich meint er - es ist leider ausnahmsweise alles etwas dunkel formuliert -, dass sich der Hochmeister hier selbst damit gleichsam der Propaganda - so wird man Hartmut Boockmanns Argumentation verstehen und übersetzen müssen - überführen würde, da er schließlich die Existenz von Kirchen im Brief - und damit die erfolgreiche Christianisierung, wie er wohl meint - konzediert habe. Boockmann deutet zudem an, dass der Orden damit zugebe, diese einem ärmlichen Wohnhause gleichenden Kirchen aufgrund ihrer einfachen Architektur zu zerstören. ${ }^{16}$ Dies ist m.E. jedoch alles eine zu moderne Perspektive auf den Brief, die nicht zur mittelalterlichen Gedankenwelt passt. (Es wäre dann zudem ein unerklärlicher Lapsus, der in einem offenen Brief, der sorgfältig - so wird man annehmen dürfen - konzeptioniert worden ist, nicht zu vermuten ist. Man wäre von polnisch-litauischer Seite auch sicherlich häufiger darauf zurückgekommen.)

Der Umstand, dass der Hochmeister ärmliche Gebäude wahrgenommen hat und deren Existenz offen zugibt, dürfte vielmehr dafür sprechen, dass von Konrad und dem Orden hier eben ein Beleg der westlichen Öffentlichkeit präsentiert werden sollte, dass die Litauer tatsächlich nur scheinbare Christen sind. Nur anständig ausgestattete Kirchen, so wird man den Brief des Ordens ex negativo besser verstehen, hätten die wahre Konversion der Litauer für den Hochmeister wohl eher belegt. Die gesehenen Gebäude fallen für den Hochmeister eben nicht in die Kategorie Kirche, sondern wurden eher als Zerrbild eines Gotteshauses wahrgenommen - wie die Litauer damit offensichtlich

15 Codex diplomaticus Prussicus. Urkunden-Sammlung zur ältern Geschichte Preussens aus dem Königl. Geheimen Archiv zu Königsberg nebst Regesten, Bde 4-6. Hg. Johannes Voigt. Königsberg 1853, 1857, 1861 [ND Osnabrück 1965], 5, Nr. CXXXV (lateinisch) vom 23. April 1403 (= OF 3, S. 41-43); 6, Nr. CXLVI (deutsche Fassung) vom 3. Mai 1403 (= OF 3, 44, 49-51).

16 Vgl. Boockmann, H.: Falkenberg, S. 78-79. Vgl. auch Rüther, Andreas: Geheimdiplomatie - Schauprozess - Medienkrieg. Polen-Litauen und der Deutsche Orden um die Zeit des Konzils von Konstanz (1414-1418). In: Bulletin der Polnischen Historischen Mission 8, 2013, S. 43-74; Kubon, S.: Außenpolitik, S. 143; DERS.: Sallinwerder, S. 328. 
Zerrbilder von wahren Christen für Konrad waren. Offensichtlich war dies ein Argument, von dem der Orden annahm, dass es auch unmittelbar plausibel für die Zielgruppe des Briefes war. Die Ordensritter hatten vielleicht nicht zuletzt die Kirchen aus ihren Herkunftsregionen im Kopf. Diese Unterschiede führten möglichweise dazu, dass die Ordensritter die kleinen litauischen Kirchen nicht ernst nahmen, sondern eben als Beleg für eine nicht, nur unvollständig und/oder scheinbar erfolgte Konversion. Hier dürfte die tatsächliche Wahrnehmung des Ordens durchscheinen und eben nicht Propaganda wider besseres Wissen. Angesichts des weiterhin kämpferischen und wankelmütigen Verhaltens der Litauer, besonders der Samaiten und von Vytautas - mal ganz abgesehen davon, welche Rolle der Orden als Auslöser für dieses Verhalten gespielt hat -, war eine solche Auffassung aus der Ordensperspektive im Rahmen der Gesamtsituation sicher auch nicht komplett abwegig.

Es war schließlich nicht zuletzt Vytautas, der viel zu dieser verwirrenden Situation beigetragen hat. Er war nicht nur der Cousin des polnischen Königs, sondern auch dessen größter Rivale. ${ }^{17}$ Er versuchte, eine eigene Herrschaft von der Ostsee bis zum Schwarzen Meer zu etablieren. Dafür schloss und brach er Verträge, wie es ihm gerade passte. ${ }^{18}$ Dies ging nicht selten mit einem Wechsel der Religion einher. Er ließ sich fünfmal taufen (mal griechisch mal lateinisch) und zuweilen kehrte er zum Heidentum zurück. Hochmeister Konrad von Jungingen hatte also zumindest plausible Gründe, warum er eine endgültige Konversion von den Litauern und insbesondere von Vytautas und den Samaiten bezweifelte. Auch die ,offizielle“ Christianisierung durch die Errichtung der polnisch-litauischen Union blieb in der Auffassung des Ordens dadurch offensichtlich mehr als nur zweifelhaft. Es war für den Orden um 1400 eine offene Situation und erst ex eventu konnte man erkennen, dass dieses Mal der Übertritt zum Christentum endgültig gewesen sein sollte.

Es waren vor allem das gegenwärtige Verhalten, aber auch die vergangenen Taten von Vytautas, die schließlich maßgeblich waren für die Wahrnehmung der Litauer durch den Orden. Der Orden erinnerte sich nur zu gut, dass Vytautas Verträge mehrere Male gebrochen, Verrat begangen und dies häufig mit einer Rückkehr ins Heidentum verbunden hatte. Eine Stelle in der Chronik des Johann von Posilge zum Jahr 1409 zeigt den Zusammenhang von Erinnerung und Wahrnehmung sehr deutlich: ${ }^{19}$ Derselbe herczog Wytold von angeborner bosheit ist eyn vorreter, und dy heylige cristinheit an des ordins lande und lute nu czum virden mole jemerlichin hat vorrothin, und der cristinheit und dem ordin grosin schadin hat czugeczogin, und hat nort eynen cristinlichin schin. Hier wird Vytautas vorgeworfen, ein Verräter zu sein und nun schon vierfachen Verrat - gemeint ist der Bruch von

17 Die treffendste Charakterisierung findet sich bei Boockmann, H.: Orden, S. 154 bzw. Ders.: Falkenberg, S. 59-60.

18 Vgl. die Aufstellung bei Kubon, S.: Sallinwerder, S. 309-310.

19 Johanns von Posilge, Officials von Pomesanien, Chronik des Landes Preussen. In: Scriptores rerum Prussicarum. Die Geschichtsquellen der preussischen Vorzeit bis zum Untergange der Ordensherrschaft. Bd. III. Hg. v. Theodor Hirsch - Max Töppen - Ernst Strehlke. Leipzig 1866. S. 79-388, hier S. 307. Zu dieser Stelle und seinem Kontext siehe Kubon, S.: Sallinwerder, S. 309-310. Vgl. zu Chronik und Verfasser Mentzel-Reuters, Arno: Unde den vride machten wider in dem lande - Kriegsziele in der Historiographie des Deutschen Ordens. In: Ordines Militares. Yearbook for the Study of the Military Orders 18, 2013, S. 81-103. 
vier Friedens- bzw. Waffenstillstandsverträgen mit dem Orden - verübt zu haben. Er sei daher nur ein scheinbarer Christ. Es ist besonders zu betonten, dass diese Stelle einem offenen Brief des Ordens entstammt. Johann von Posilge hat nur wenige Änderungen vorgenommen, als er dieses Dokument in seine Chronik inserierte. ${ }^{20}$ Hier aber sehen wir nun sehr deutlich den Zusammenhang zwischen Erinnerung, zeitgenössischer Geschichte und der Wahrnehmung gegenwärtiger Ereignisse.

Der eingangs schon angeführte Brief von 1396 ist hingegen die wichtigste Quelle, die den Zusammenhang zwischen Wahrnehmung und Erinnerung belegen soll. Die maßgebliche Stelle sei daher hier noch einmal wiederholt, da sie in ihrer Bedeutung gar nicht überschätzt werden kann: ${ }^{21}$ Dorumb, das got nicht gebe, ab eyn umslag geschege, als vor ofte gescheen ist, von im und sienen vorvarn, als von konig Mindow von Littowen, das sich der orden sien deste bas mochte dirweren des umslages adir abkerunge. Sie zeigt eben ganz deutlich, dass die Erinnerung an den Rückfall ins Heidentum durch König Mindaugas selbst um 1400 noch aktiviert werden konnte. Modern-didaktisch gesprochen finden wir hier einen Beleg für das im Ordensland herrschende Geschichtsbewusstsein (Jeismann). Dem Orden war auch jetzt um 1400 überdeutlich präsent, was ca. 150 Jahre zuvor geschehen war. Offensichtlich hatte diese Erfahrung auf den Orden einen großen Eindruck gemacht. Der Orden kann daher als Erinnerungsgemeinschaft bezeichnet werden. Die Erinnerung an den regelmäßig vergeblichen Kampf gegen das litauische Heidentum, an den wiederholten Abfall vom Christentum auch in früheren Zeiten, hatte dann eben einen großen Einfluss auf die Wahrnehmung der zeitgenössischen Litauer, deren Wankelmütigkeit ein übriges zur aktuellen Situationsdeutung tat. Diese Erinnerung hat immer noch die gegenwärtige Wahrnehmung der Litauer als vorgebliche Christen und ,unsichere Kantonisten‘ bestimmt. Vytautas‘ Verhalten der Gegenwart ebenso wie das Verhalten von Mindaugas in der Vergangenheit strahlte dann eben auf alle Litauer und die polnisch-litauische Union ab. Dafür ist der angeführte Brief der Kronzeuge. Wichtig ist zu betonen, dass dieser Brief eben kein offener Brief war, sondern an andere Amtsträger des Deutschen Ordens gerichtet war. Es ist höchst unwahrscheinlich, dass wir es hier mit einem verzerrten Bericht zu tun haben, sondern mit der kaum gefilterten Wahrnehmung des Hochmeisters.

Um es kurz zusammenzufassen: Es scheint sehr wahrscheinlich zu sein, dass diese Quellen die Auffassung stützen, dass die zeitgenössische Beschreibung der Litauer als vorgebliche Christen, relapsii oder Heiden durch den Deutschen Orden unter Hochmeister Konrad von Jungingen seine tatsächliche Wahrnehmung widerspiegelt und eben nicht als Propaganda wider besseres Wissen um der territorialen Expansion willen zu verstehen ist. Der Orden erinnerte sich schließlich immer noch an den Rückfall von König Mindaugas und andere namentlich nicht genannte Vorfahren von Vytautas, der zudem auch eben nicht den beständigsten Eindruck machte, um es vorsichtig zu formulieren. (Eine Differenzierung der verschiedenen (polnisch-)litauischen Akteure durch den Orden, die ja hätte vorgenommen werden können, fand offensichtlich nur partiell

20 Die Übernahmen sind in der Edition ausgewiesen. Vgl. die Hinweise der Herausgeber in den Anmerkungen, Johann von Posilge, S. 306, Anm. 2; S. 304, Anm. 1, und in der Einleitung, S. 39-40.

21 BGDO I, Nr. 246. 
statt.) Die Erinnerungen sowohl an zeitgenössische als auch an historisch weit entfernte Ereignisse waren es, die daher dann die Wahrnehmung der gegenwärtigen Situation offensichtlich stark lenkten und die daraus folgende Politik bestimmten. Unter diesen Umständen war es auch ca. 15 Jahre später noch schwer für den Orden, die politisch-religiösen Änderungen durch die polnisch-litauische Union für dauerhaft zu begreifen. Andere Handlungsoptionen zu entwickeln, um mit diesen Änderungen umzugehen, war unter dieser Umständen zwar nicht unmöglich - man muss im Hinterkopf behalten, dass auch Preußen zuerst nur ein alternatives Handlungsfeld zum Heiligen Land war -, aber offensichtlich doch nicht sehr wahrscheinlich, schließlich sah man im Orden offensichtlich keine Änderungen, sondern ein immer gleiches Handeln der Litauer über Jahrhunderte hinweg. Dazu trug sicherlich bei, dass die territoriale Herrschaft des Ordens um 1400 als konsolidiert beschrieben werden kann. Das war dann offensichtlich keine politische Rahmung, die dazu einlud, auch zarte bis deutlichere Hinweise auf eine Änderung der politischen Großwetterlage, die es neben dem wankelmütigen Verhalten von Vytautas schließlich zur Genüge gab, wahrzunehmen und darauf anders als bisher zu reagieren.

Welche Erklärungsmöglichkeiten für diese Wahrnehmung des Hochmeisters und des Ordens bieten nun die Theorien der kulturwissenschaftlichen Gedächtnisforschung? Diese hält theoretisch zahlreiche sehr elaborierte Ansätze, die u.a. mit den Namen Pierre Nora und Aleida und Jan Assmann verbunden sind, bereit. ${ }^{22}$ An dieser Stelle kann es jedoch nur darum gehen zu zeigen, dass sich für die hier beschriebenen Umstände die Rezeption der klassischen Ansätze von Maurice Halbwachs weiterhin lohnt, da seine Theorie des kollektiven Gedächtnisses im Zusammenhang mit der von ihm beschriebenen sozialen Bedingtheit individueller Erinnerung unmittelbare Erklärungsmöglichkeiten zu bieten scheint. ${ }^{23}$ Es kann aber hier nur das Potential dieser theoretisch ausgesprochen voraussetzungsreichen Ansätze auch in der Ordenshistoriographie ausgelotet werden, um zu prüfen, ob sich die Beschäftigung in extenso lohnen könnte hinsichtlich des Zusammenhangs von Wahrnehmung, Erinnerung und Politik in Bezug auf die Kämpfe des Deutschen Ordens gegen die Litauer.

Es muss zunächst besonders die Erkenntnis von Halbwachs unterstrichen werden, dass jede Gesellschaft ein Gruppengedächtnis hat, das so genannte kollektive Gedächtnis. Zu diesem kann man allerdings nur über das individuelle Gedächtnis einen Zugang finden. Für unsere Fallstudie ist das Paradebeispiel der Brief, in dem an den Abfall von Mindaugas erinnert wird. Hier findet sich über die individuelle Erinnerung ein Zugang

22 Aufgrund seiner Komplexität übergehe ich hier das Theorieprogramm von Aleida und Jan Assmann und insbesondere ihren Ansatz des kulturellen Gedächtnisses. Ebenso müsste man Pierre Noras Ansatz der Erinnerungsorte auf seine Fruchtbarkeit für die hier zu untersuchende Fragstellung prüfen. Ein Überblick zu diesen und weiteren wichtigen Konzepten findet sich bei ErLl, A: Gedächtnis, bes. S. 11-34. Hier ist auch die entsprechende Literatur vollumfänglich verzeichnet.

23 In Erlls Zusammenfassung findet sich Halbwachs Werk in nuce mit den für uns wesentlichen Potentialen und Defiziten zusammengefasst; vgl. ibidem, S. 11-16; danach hier. Wesentliche Anknüpfungspunkte finden sich bereits in Halbwachs' erster großen Studie zu dem Thema aus dem Jahr 1925 (HALbwachs, M.: Gedächtnis), in der er die soziale Bedingtheit von Erinnerung („cadre sociaux“) in den Mittelpunkt stellt. Die Ergebnisse der weiteren Bearbeitung finden sich dann in DERs.: Kollektive Gedächtnis (zur Editionsgeschichte und den zuverlässigen Ausgaben bzw. Übersetzungen siehe auch ERLL, A.: Gedächtnis, S. 11-16). 
zum kollektiven Gedächtnis. Zwar könnte man hier natürlich einwenden, wie individuell ein Brief denn überhaupt sein kann, der durch eine Kanzlei gelaufen ist? Aber vielleicht würde dieser Einwand doch vielmehr auf das Wechselspiel von individuellem und kollektivem Gedächtnis hinweisen, das schon Halbwachs betont hat: „Man kann ebenso gut sagen, daß das Individuum sich erinnert, indem es sich auf den Standpunkt der Gruppe stellt, und daß das Gedächtnis der Gruppe sich verwirklicht und offenbart in den individuellen Gedächtnissen. "24 Genau das dürfte eben in einem ,Verwaltungsakt" vonstatten gegangen sein, an dessen Ende ein solcher Brief die Kanzlei verlässt.

Das kollektive Gedächtnis nimmt, wie Halbwachs betont, mittels Interaktion, Kommunikation, Medien und Institutionen auf die Vergangenheit Bezug. Dieses Gedächtnis ist dabei - und nun wird es für den Deutschen Orden besonders interessant - abhängig von den so genannten „cadres sociaux“ bzw. dem sozialen Bezugsrahmen, in dem eine Gruppe in einer Gesellschaft verortet ist. ${ }^{25}$ Halbwachs betont, dass jedes menschliche Wesen zu zahlreichen verschiedenen Gruppen wie Familie, Religion und Beruf gehört und damit unterschiedliche Erfahrungshorizonte hat. Die spezifische Kombination dieser Gruppenzugehörigkeiten und ihrer Erinnerungsformen mache dann das individuelle Gedächtnis aus. Dies dürfte der wesentliche Punkt und die Erklärung dafür sein, warum das kollektive Gedächtnis des Ordens so dezidiert bestimmte, dass die Litauer in Preußen weiterhin als Heiden wahrgenommen werden mussten. Die Ordensritter waren hinsichtlich dieser Gruppenzugehörigkeiten wie Familie, Religion und Beruf (sogar im ursprünglichen Sinne des Wortes) natürlich nur wenig heterogen, um es vorsichtig auszudrücken. Der Deutsche Orden kann daher in seiner Ausprägung als Erinnerungsgemeinschaft als eine Art „totale Organisation“ bezeichnet werden. Das kollektive Gedächtnis des Ordens ist daher sehr statisch, da nur wenige Friktionen zwischen den verschiedenen, recht homogenen Gedächtnissen der Ordensmitglieder bestanden. Das wiederum führt zu einem äußerst konservativen Gedächtnis, das nicht sehr offen dafür ist, neue Erfahrungen oder Umstände als neue Erfahrungen und Umstände auch wahrzunehmen. Vielmehr wurden auch neue Situationen von allen oder zumindest den meisten Gruppenmitgliedern als altbekannte Erfahrungen wahrgenommen. Das betrifft dann auch die Wahrnehmung der Litauer durch den Orden, die man durch die Schablone der Erinnerung als immer schon wankelmütig wahrnahm.

$\mathrm{Zu}$ einer solchen Situation trägt dann auch der Umstand bei, dass das kollektive Gedächtnis ohnehin in jedem Fall sehr selektiv und rekonstruktiv arbeitet. Es ist schließlich wichtig für den Aufbau einer Identität. ${ }^{26}$ Es erinnert, was zur Selbstwahrnehmung und den Primärinteressen der Gruppen passt. Auch dieser Umstand zeigt, dass dieser theoretische Ansatz eine gute Erklärung bieten kann, warum es für den Deutschen Orden in Preußen um 1400 schwierig war, angemessen auf die geänderten Umstände zu

24 Halbwachs, M.: Gedächtnis, S. 23.

25 Ibidem, passim.

26 Vgl. Halbwachs, M.: Kollektive Gedächtnis, nach Erll A.: Gedächtnis, S. 14. Aufgrund der beschränkten Zugänglichkeit von Bibliotheken und Literatur in den Zeiten der Corona-Pandemie (März 2020) konnten manche Aspekte, wie hier, nicht noch einmal im Original verifiziert, sondern mussten aus sekundären Bearbeitungen übernommen werden. 
reagieren. Das kollektive Gedächtnis erinnerte den Heidenkampf im Baltikum und die bisher gescheiterte Christianisierung der Litauer so stark, dass der Hochmeister die zeitgenössischen Änderungen im Rahmen der polnisch-litauischen Union als fundamentale Veränderung der Gesamtsituation gar nicht wahrnehmen konnte und weiterhin auf die Stiftungsaufgabe des Ordens, den Heidenkampf, pochte. Diese war schließlich zentral für die Gruppenidentität in einem geistlichen Ritterorden.

Es ist also insgesamt mehr als nur wahrscheinlich, dass nicht nur gegenwärtige Ereignisse, sondern auch oder besser: vor allem die Erinnerungen an solche der weit vergangenen Zeiten die Wahrnehmung der Litauer durch den Orden präfiguriert und die daraus folgende Politik des Hochmeisters bestimmt haben. Der ordensinterne Charakter der einschlägigen Briefe dürfte ein wesentliches Argument dafür sein, dass man hier tatsächlich die Wahrnehmung des Hochmeisters erkennen kann. Unter diesen Umständen konnte Konrad von Jungingen die Litauer dann nur als vorgebliche Christen, ganz wie ihre Vorfahren, beschreiben. Das latente Geschichtsbewusstsein des Hochmeisters hat sich hier Bahn gebrochen. ${ }^{27}$ Diese Wahrnehmung wurde zudem gestützt durch die Kurfürsten, wie auch durch die Teilnehmer der Litauenreisen aus dem ganzen westlichen Europa, die bis in die 1420er Jahre diese kontinuierlich fortgesetzt haben. ${ }^{28}$ Auch hier machte man weiter wie bisher im Umgang mit den Litauern und betrachtete sie als Heiden, die zu bekämpfen waren. Wie hätte man in Preußen angesichts des Verhaltens der tragenden Säulen der Ordensunterstützer auch seine Politik gänzlich ändern können sollen?

Es gab zwar, und das muss abschließend erwähnt werden, auch prominente und weniger prominente Stimmen, die dem Orden nahgelegt haben, sein Verhalten zu ändern angesichts einer neuen Situation. Die berühmten und immer wieder angeführten Verbote der Litauenreisen durch den römisch-deutschen König Wenzel 1395 und Papst Bonifaz IX. 1403 blieben allerdings ziemlich wirkungslos und wurden ordensintern kaum diskutiert - zumindest hat sich das weniger in den schriftlichen Quellen niedergeschlagen als man es angesichts dieser bedeutenden Schritte hätte erwarten können. Die Verbote wurden wohl einerseits für zu parteiisch und erschlichen betrachtet und vor allem andererseits für zu absurd gehalten angesichts des tagesaktuellen Verhaltens der Litauer. Diese Verbote konnten daher die Wahrnehmung der Litauer durch den Orden nicht ändern. ${ }^{29}$ Allerdings war die Auffassung des Ordens auch im Preußenland selbst nicht unwidersprochen. Dies belegt die Diskussion der Jahre 1406 und 1407 um das Schalwenkorn und das Wartgeld, das zur Bewachung der schalauischen Grenze erhoben wurde. Dies wollten die Ritter und Knechte des Landes nicht mehr bezahlen, da die Litauer ja

27 Im Übrigen stützt aber auch die Persönlichkeitsstruktur von Konrad, die sich in seiner Außenpolitik spiegelt, eine solche Deutung: Konrad neigte zu ad-hoc-Reaktionen und nicht zu weitreichender Planung in der Außenpolitik und war ein Getriebener statt Antreiber. Er nutzte Routinen zur Entscheidungsfindung, wie das strikte Beharren auf der Einhaltung von Verträgen etc.; Kubon, S.: Außenpolitik, S. 328-329. Zu diesen Mechanismen in Entscheidungsprozessen würde es auch passen, ,Erinnerungsroutinen“ als Entlastung in komplexen Situationen zu nutzen.

$28 \mathrm{Zu}$ den Preußenreisen und ihrer zeitlichen Ausdehnung vgl. PARAvicini, W.: Preussenreisen. Anmerkungen zur Rolle der Kurfürsten bei KuBon, S.: Grundzüge, S. 94 und DERS.: Außenpolitik, S. 85, 89, $102,103$. 
nun bezwungen seien. (Das deutet die Auffassung von einer Christianisierung der Litauer nur an, beinhaltet sie aber sicherlich.) Nur auf eindringliche Bitte des Hochmeisters wurde das Geld doch weiterhin gezahlt. Auch dieser Umstand löste beim schon kränkelnden Konrad von Jungingen, der Ende März 1407 sterben sollte, keine nachhaltige Erschütterung aus. Vielmehr zeigt diese Episode, dass Konrad tatsächlich immer noch mit der Gefahr aus Litauen rechnete. ${ }^{30}$

Das Konzept des kollektiven Gedächtnisses und der sozialen Bedingtheit des individuellen Gedächtnisses nach Maurice Halbwachs dürfte es schließlich sein, das diese Situation dann eben plausibel und theoriegeleitet erklären kann: Der Orden war letztlich in ihrer Funktion einer Erinnerungsgemeinschaft eine „totale Institution“. Alle Mitglieder hatten sehr ähnliche Grundlagen der Erinnerung, sodass vor diesem Hintergrund andere Auffassungen es schwer hatten, sich durchsetzen zu können. Das erklärt, warum sich die Situationsauffassung des Ordens euphemistisch als ,altbewährt' beschrieben lässt und warum aufgrund der erinnerungsgeleiteten Wahrnehmung andere Stimmen, die Einwände erhoben, kein Gehör finden konnten. Letztlich läuft bei der Erklärung des Verhaltens des Hochmeisters in der Politik gegenüber Litauern alles auf die berühmte Morgenstern'sche Logik hinaus: „weil, so schließt er messerscharf, nicht sein kann, was nicht sein darf ..." - nur ohne dass dies eine bewusste Entscheidung war, sondern in der Reaktivierung der Erfahrungen des kollektiven Gedächtnisses und im Geschichtsbewusstsein begründet lag.

Es muss unterstrichen werden, dass diese Erkenntnisse vorerst nur auf die Zeit von Konrad von Jungingen (1393-1407) angewandt werden können. Es wäre notwendig, auch die Regierungszeiten der anderen Hochmeister um $1400 \mathrm{zu}$ untersuchen. ${ }^{31}$ Es ist sehr wahrscheinlich, dass bspw. Hochmeister Michael Küchmeister (1414-1422) ${ }^{32}$ die Litauer schon anders wahrgenommen hat als Konrad. Generell wäre es überdies wichtig, sich nicht nur auf schriftliche Quellen zu stützen. Es müsste gefragt werden, ob auch andere Medien existierten, die der Wahrnehmung der Litauer als vorgebliche Christen Nahrung gaben. Was signalisiert z.B. die wehrhafte Architektur und die Ausstattung der Ordensburgen so z.B. die Marienburg? Wie sind Rituale und Performanzen - z.B. die Tischlesungen im Orden, der Ehrentisch nach erfolgter Reise sowie die Ritterschläge im Rahmen der Kriegszüge - in diesem Kontext zu interpretieren?

Eine Menge Fragen müssen daher hier offenbleiben. Alles in allem handelte es sich schließlich nicht zuletzt um eine erste Skizze des Potentials der Zugänge der kulturwissenschaftlichen Erinnerungsforschung und um ein Plädoyer für ihre Anwendung auf die Geschichte des Deutschen Ordens. Dabei sollte man sich natürlich nicht nur auf die Studien von Maurice Halbwachs beschränken, sondern auch vor allem die Schriften von Aleida und Jan Assmann hinzuziehen. Es wäre zudem aber auch die Applikation

30 Johann von Posilge, S. 284-285; vgl. Kubon, S.: Außenpolitik, S. 186.

31 Hinweis darauf schon bei Kubon, S.: Grundzüge, S. 94, Anm. 38.

32 Zum Forschungstand der Außenpolitik Michael Küchmeisters siehe Kubon, S.: Hochmeister Michael Küchmeister und die Konflikte des Deutschordenslandes Preußen mit Polen-Litauen 1414-1422: Krisen ohne Ende oder eine Phase der Konsolidierung? Vorüberlegungen zu einer Studie mittelalterlicher Außenpolitik. In: Bulletin der Polnischen Historischen Mission 11, 2016, S. 359-379. 
der aktuellen politikwissenschaftlichen Theorie des Framing denkbar. ${ }^{33}$ Eine vertiefte Untersuchung mit einer breiteren Basis an Quellen aus unterschiedlichen Medien und einem erweiterten und tiefenscharfen theoretischen Überbau mit einer trennscharfen Verwendung der relevanten wissenschaftlichen Begrifflichkeiten dürfte sich lohnen und wäre sicherlich reizvoll, um alte Gewissheiten weiter und vertieft in Frage zu stellen und um endlich tragfähige und überzeugende Erklärungen bzgl. der Ausgangsfragestellung zu erlangen. ${ }^{34}$

\section{Pohled řádu německých rytířů na Litevce kolem roku 1400 a význam kolektivní paměti}

Politiku řádu na počátků 15. století spoluovlivňovaly logicky jak soudobé zkušenosti velmistra Konráda z Jungingenu (1393-1407) a dalších představitelů řádu německých rytírư s jednáním Litevců a Žmudínů a aktuálními Vytautasovými postoji, tak také vzpomínky na dřivější činy Litevců pod Mindagausovou vládou. Na základě některých obratů v listech velmistra Konráda a kronice Jana z Posilge lze přesvědčivě doložit, že existovala úzká souvislost mezi pamětí, vnímáním a politikou. Řád vnímal Litevce kolem roku 1400 stále jako pohany, s nimiž je třeba bojovat a které je třeba christianizovat. Tento postoj nelze ovšem hodnotit jako propagandu, nýbrž jako reálný pohled řádu na Litevce. Srozumitelné vysvětlení tohoto překvapivého jevu nabízí vědecké teorie o vytváření paměti. Článek vychází ze základních myšlenek klasických studií z pera Maurice Halbwachse o kolektivní paměti a „sociálním rámci“ („cadres sociaux“), resp. o sociálních předpokladech vytváření paměti. Ty skýtají potřebný potenciál pro výzkum situace na řádovém území v Prusku. Kromě jiného pomáhají nalézt odpověd’ na otázku, proč se pruské „rejzy“ a boje konaly ještě patnáct let po vzniku Polsko-litevské unie a „oficiální“ konverzi Litvy.

33 Wehling, Elisabeth: Politisches Framing. Wie eine Nation sich ihr Denken einredet - und daraus Politik macht. Köln 2016.

34 Zur Geschichte der Wahrnehmung anderer Religion sollte man dann vergleichsweise auch die elaborierten Forschungen von Hans-Werner Goetz heranziehen zu Früh- und Hochmittelalter. Als erster Ansatzpunkt vgl. GOETz, Hans-Werner: Die Wahrnehmung anderer Religionen und christlich-abendländisches Selbstverständnis im frühen und hohen Mittelalter (5.-12. Jahrhundert). Berlin 2013. 